\title{
On-orbit Calibration and Performance of S-NPP VIIRS DNB
}

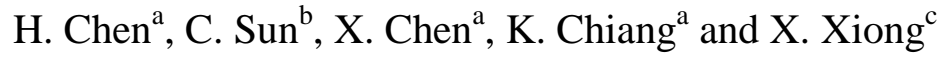 \\ ${ }^{a}$ Systems \& Applications, Inc., 10210 Greenbelt Rd, Lanham, MD 20706; \\ ${ }^{\mathrm{b}}$ Global Science Technology, Inc., 7855 Walker Dr, Greenbelt, MD 20770; \\ ${ }^{c}$ Science Systems and Exploration Directorate, NASA/GSFC, Greenbelt, MD 20771
}

\begin{abstract}
The S-NPP VIIRS instrument has successfully operated since its launch in October 2011. The VIIRS Day-Night Band (DNB) is a panchromatic channel covering wavelengths from 0.5 to $0.9 \mu \mathrm{m}$ that is capable of observing Earth scenes during both day and nighttime orbits at a spatial resolution of $750 \mathrm{~m}$. To cover the large dynamic range, the DNB operates at low, mid, or high gain stages, and it uses an onboard solar diffuser (SD) for its low gain stage calibration. The SD observations also provide a means to compute gain ratios of low-to-mid and mid-to-high gain stages. This paper describes the DNB on-orbit calibration methodologies used by the VIIRS Characterization Support Team (VCST) in supporting the NASA earth science community with consistent VIIRS sensor data records (SDRs) made available by the Land Science Investigator-led Processing Systems (SIPS). It provides an assessment and update of DNB on-orbit performance, including the SD degradation in the DNB spectral range, detector gain and gain ratio trending, stray light contamination and its correction. Also presented in this paper are performance validations based on earth scenes and lunar observations.
\end{abstract}

Keywords: VIIRS, Day-Night Band, DNB, S-NPP, Calibration, VCST

\section{INTRODUCTION}

The Visible Infrared Imaging Radiometer Suite (VIIRS) on-board the Suomi National Polar-orbiting Partnership (SNPP) spacecraft was launched on October 28, 2011 with successful operation for more than four years. VIIRS was activated on November 8, 2011. Following a series of functional and early orbit tests, its nadir door opened on November 21, 2011. Since then, Earth View (EV) data have been collected for all the reflective solar bands (RSB). The thermal emissive bands (TEB) became operational after January 18, 2012 when the passive cryo-cooler door was opened. The VIIRS observations are made in 22 spectral bands, including 16 moderate resolution bands, 5 imaging bands, and one panchromatic Day-Night band (DNB), with wavelengths ranging from 0.41 to $12 \mu \mathrm{m}$. The calibrated and geo-located radiances and reflectance/brightness-temperature are Sensor Data Records (SDRs), which are used to generate the Environmental Data Records (EDRs). VIIRS was built and operated with a strong heritage from the Moderate Resolution Imaging Spectroradiometer (MODIS) on-board the Aqua and Terra spacecrafts. The experience from MODIS, including its design, spectral band coverage, on-board calibrators (OBCs), and calibration approaches and strategies, has been beneficial in the subsequent operation of the VIIRS instrument.

The VIIRS OBCs include a flat panel v-grooved blackbody (BB), a solar diffuser (SD) and a SD stability monitor (SDSM) that tracks any SD on-orbit degradation. As shown in Figure 1, the rotating telescope (RTA) design was chosen rather than a scan-mirror in order to suppress stray light. The RTA is a three-mirror anastigmatic design such that light out of the RTA reflects onto a mirror that rotates at half the angular velocity of the RTA, thus called the half-angle mirror (HAM). As RTA rotates, it takes a view of three calibration sectors: The space view (SV) points out to space just above the mesospheric limb, and occurs just before the start of the earth view (EV); the BB view occurs after the RTA sweeps across the Earth and is inside the instrument; and the SD illuminated by the sun through a solar attenuation 
screen during a 2 to 3 minute period each orbit. The SV is an aperture providing a view of cold space to allow for a zero radiance input signal on each scan. The Moon can also be observed via the SV at a frequency of approximately once per month.

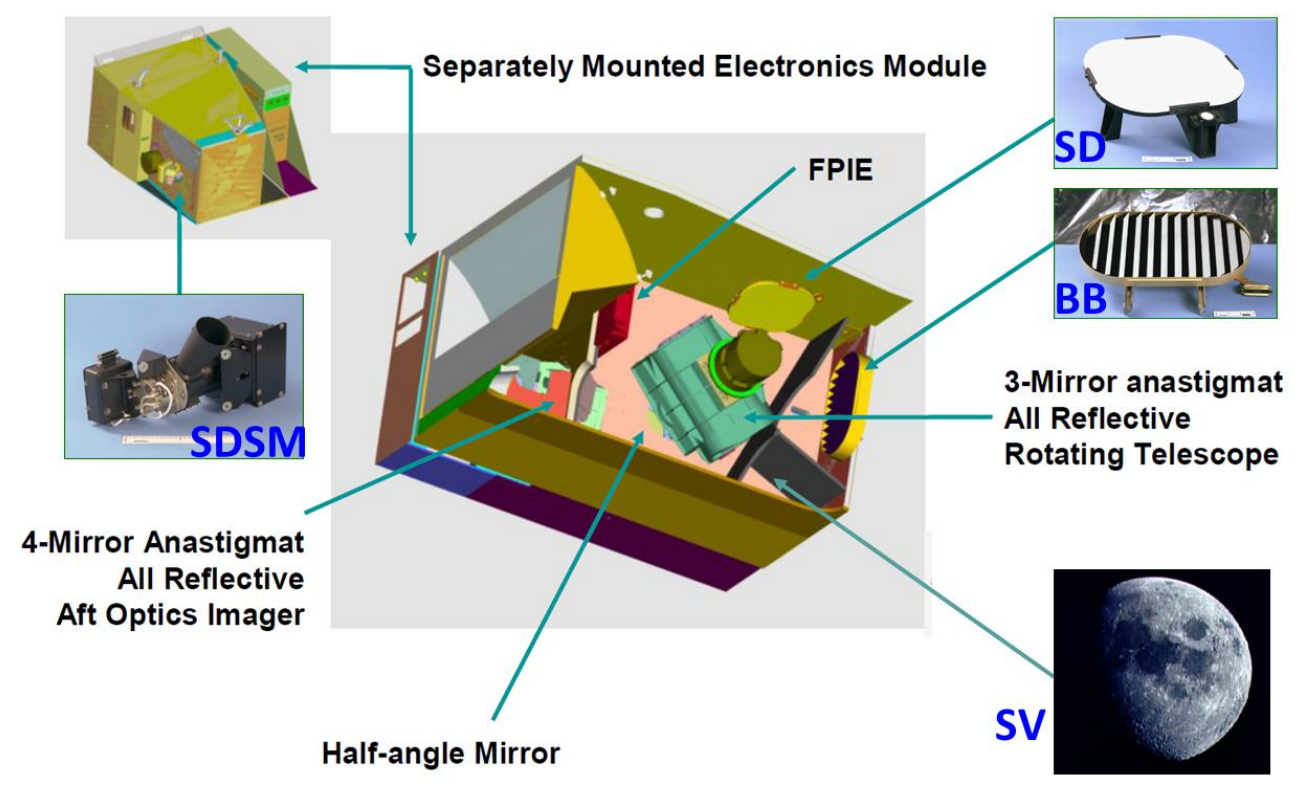

Figure 1 S-NPP VIIRS Overview with On-orbit Calibrators.

The DNB is a panchromatic visible/near-infrared (Vis/NIR) band designed to detect radiance from the brightest daytime scenes down to very dim nighttime scenes illuminated by a quarter moon. Its dynamic range spans over 7 orders of magnitude. As presented in Table 1, the DNB has a wide spectral bandpass, centered at $700 \mathrm{~nm}$. The DNB sensor is a temperature-controlled charge-coupled device (CCD). The CCD focal plane array (FPA) is sectioned into 4 stages, where each stage essentially functions as a separate imager, though they all reside on the same FPA. Using 32 aggregation modes, DNB compensates for perspective and foreshortening in a way that results in an almost constant 750 $\mathrm{m}$ ground resolution in all scans.

Table 1 DNB Spectral Specification

\begin{tabular}{|c|c|c|c|c|c|}
\hline $\begin{array}{l}\text { VIIRS } \\
\text { Band }\end{array}$ & $\begin{array}{c}\text { Central } \\
\text { wavelength }(\mu \mathrm{m})\end{array}$ & $\begin{array}{c}\text { Band } \\
\text { width }(\mu \mathrm{m})\end{array}$ & $\begin{array}{c}\text { Wavelength } \\
\text { Range }(\mu \mathrm{m})\end{array}$ & $\begin{array}{c}\text { Band } \\
\text { Explanation }\end{array}$ & $\begin{array}{c}\text { Spatial Resolution at } \\
\text { nadir }(\mathrm{m})\end{array}$ \\
\hline DNB & 0.7 & 0.4 & $0.5-0.9$ & Visible/Reflective & 750 across full scan \\
\hline
\end{tabular}

There are four detector arrays at three different radiometric gains used to cover the wide dynamic range. Each detector array has 672 sub-pixel detectors along-track, which are aggregated onboard to create sixteen near constant $750 \mathrm{~m}$ alongtrack pixels for each along-scan frame. The high gain stage (HGS) has two redundant arrays, identified as HGA and HGB. Each HGS array has 250 along-scan sub-pixel detectors operating in time delay integration (TDI) for nighttime imagery. The third array is identified as middle gain stage (MGS), which has 3 along-scan sub-pixel detectors operating in TDI. The final low gain stage (LGS) has a single sub-pixel detector in the scan direction.

In NOAA Interface Data Processing Segment (IDPS) calibrations, the DNB is calibrated through a monthly VIIRS recommended operating procedures (VROPs) and the SD observations [1]. Basically, there are two VROPs: VROP705 is for gain ratio determination, and VROP702 is for offset determination. The VROP data were analyzed to estimate the dark offset and cross-stage gain ratios. The drawback of using VROP is the loss of science data as the instrument in 
maneuver operations. Furthermore, nighttime airglow contamination may also be embedded in VROP data so as to introduce additional estimate uncertainty. Alternatively, the NASA VIIRS characterization support team (VCST) has proposed a DNB calibration approach that directly utilizes calibration view data to continuously track the gain and offset over time [2]. This method determines the dark offset drift using nighttime observed calibrator dark signals, which is free from airglow contamination. It estimates the cross-stage gain ratios based on simultaneously observed SD signals in each solar calibration event. It can continuously update the offset and gain ratio drift as the calibration view data is continuously collected in a repeatable orbital cycle. A time-dependent modulated RSR is applied to compute DNB gains to reflect the throughput change across the solar spectrum. Moreover, to remove nighttime airglow impacts, a pitch maneuver deep space scene is used as the HGS dark offset reference. A stray light correction is implemented to remove stray light in the affected area around the southern and northern terminators.

\section{DEGRADATION BEHAVIOR}

The VIIRS SD bi-directional reflectance distribution function (BRDF) was characterized pre-launch. The on-orbit changes of the SD BRDF are monitored by the on-board SDSM. In general, the SDSM is operated during each SD calibration to perform alternate views of the sunlight through a fixed penetration screen (Sun view), and of sunlight diffusely reflected from the SD panel (SD view). The SD on-orbit degradation can be calculated using the ratio of brightness along the two viewing paths. The center wavelength of VIIRS reflective solar bands (RSB) covers a range from $412 \mathrm{~nm}$ to $2250 \mathrm{~nm}$. Specifically, we have M1: $412 \mathrm{~nm}$; M2: $445 \mathrm{~nm}$; M3: $488 \mathrm{~nm}$; M4: $555 \mathrm{~nm}$; M5 672 nm; M6: 746 nm; M7: 865 nm; M8 1240 nm; M9: 1375 nm; M10: 1610 nm; M11: $2250 \mathrm{~nm}$. Figure 2 shows the VIIRS RSB gain degradation over a 4-year period. From the yearly degradation point of view, the case of the first year to the second year illustrates the largest degradation, especially for M7 and M8. After that, the subsequent years show similar degradation.

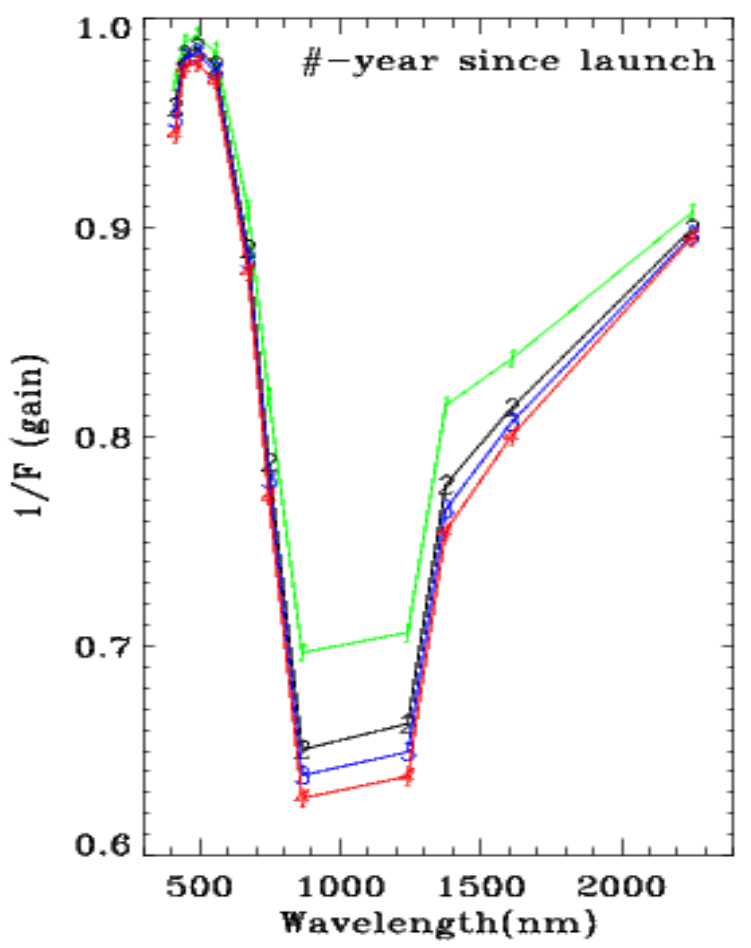

Figure 2 VIIRS RSB Gain Degradation in 4 Years. Where different colors present different year, such as first-year (GRN), second-year (BLK), third-year (BLU) and fourth-year (RED).

Due to unexpected mirror darkening, the SNPP VIIRS RTA throughput has significantly degraded on-orbit [3]. As the degradation is wavelength dependent, DNB RSR is expected to be significantly affected as it has a wide bandpass 
wavelength range. Thus, DNB radiometric gain needs to be convolved with a modulated RSR to reflect the change of incident solar radiance. In practice, the VCST calibration method uses a time-dependent modulated RSR to estimate the DNB LGS gain during solar calibration events [2]. Figure 3 shows the pre-launch RSR and modulated RSR at orbits 1201, 945, 2194, 17000 and 21200. The modulated RSRs are based on degradation parameters estimated from RSB Ffactor [4]. The orbit-945 occurs on January 3, 2012. The orbit-1201 happens on January 21, 2012, when the VIIRS SWIR focal plane temperature began to stabilize. The orbit-2194 is on March 31, 2012, when the VIIRS SWIR F-factor began to stabilize after a spacecraft control anomaly [2,3]. The orbit-17000 roughly presents the VIIRS instrument's completion of 3 years of operation, and the orbit- 21200 denotes 4 years of operation. The modulated RSR curves show that the relative response has been decreasing for wavelength higher than $~ 700 \mathrm{~nm}$, and increasing for wavelength lower than $\sim 700 \mathrm{~nm}$. The rate of change of the RSR modulation is much faster in the early mission, as it experienced rapid degradation. The difference between the year three and year four modulated RSR is very small.

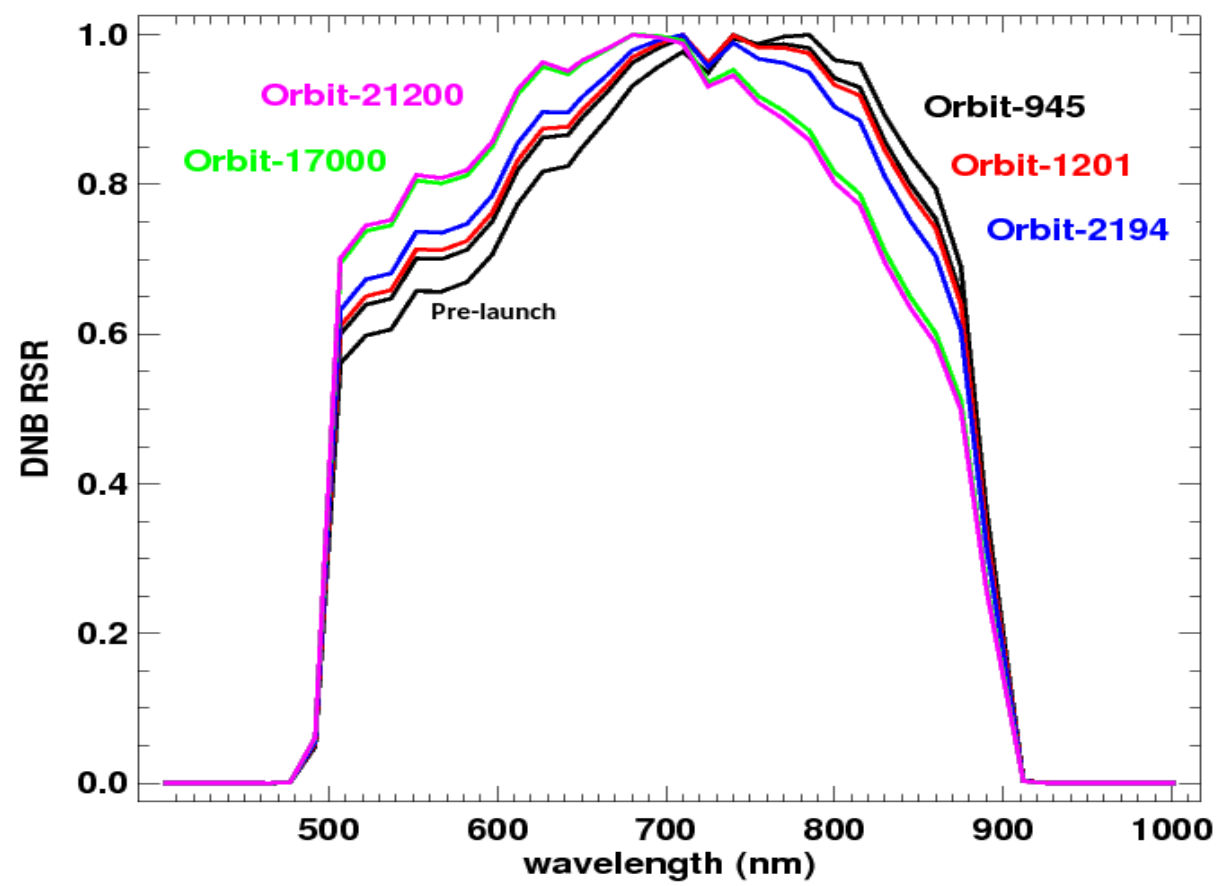

Figure 3 Pre-launch Measured and On-orbit Modulated DNB RSR.

Similar to the VIIRS RSB, the primary radiometric calibration coefficient for DNB is the F-factor, which corresponds to the inverse of the DNB gain. It is a linear multiplicative coefficient correcting the prelaunch radiometric coefficients. The principle method for determining the value of the F-factor is the use of the SD while it is fully illuminated by the Sun. An alternative method to determine the F-factor is using VIIRS observations of the Moon. The lunar F-factor acts as an independent check of the SD F-factor. Figure 4 shows the trending results of all 16 detectors in the DNB. Different colors represent different detectors for the SD 1/F (detector dependent). However, the diamond denotes the lunar 1/F in the sense of a band average. Notice that all DNB detectors have shown a similar behavior. There is an approximately $18 \%$ degradation after four years of on-orbit operations. In this figure, the band averaged lunar observations are also plotted for comparisons. The data in the first lunar event is normalized to the DNB band-averaged gain value. The result shows that the moon observations validate/match the DNB gain trending very well. 


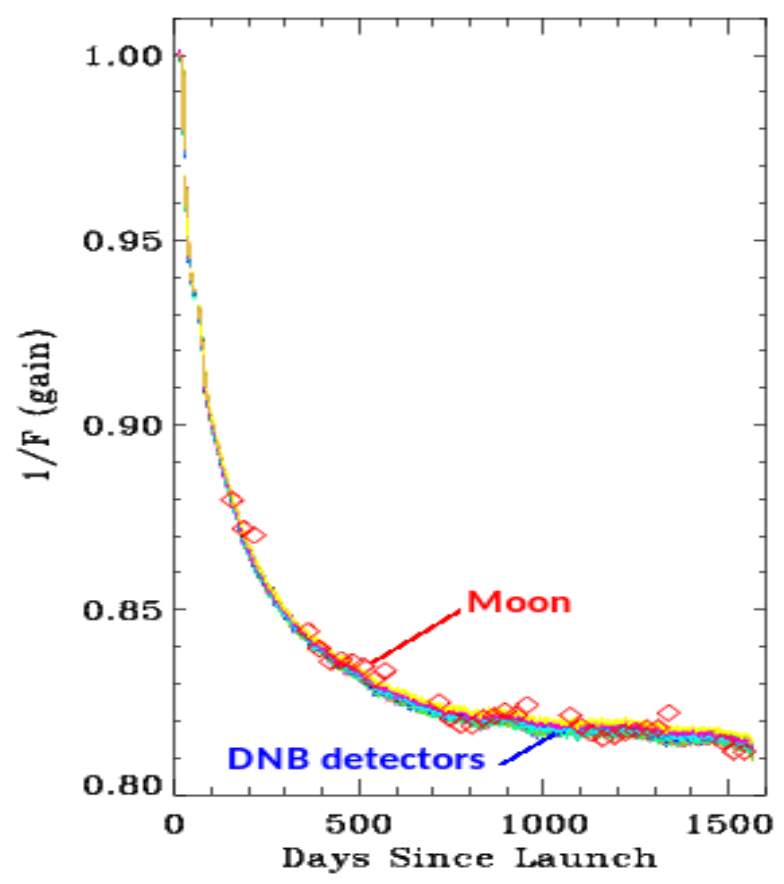

Figure 4 DNB Gain Trending via SD and Lunar Data.

\section{CALIBRATION PERFORMANCE AND TRENDING}

The VIIRS DNB is designed to make global observations day and night with a large dynamic range at three different gain stages of LGS, MGS and HGS, which has very high sensitivity at low light conditions over nighttime orbits. The SNPP VIIRS DNB calibration is performed periodically to track the on-orbit detector gain change. The LGS gain is determined from the SD measurement when the SD is fully illuminated by the Sun through an attenuation screen. Due to the high sensitivities of MGS and HGS, the fully illuminated SD signal will saturate detectors in these gain stages and thus cannot be used to compute the gains. However, carefully selected data, such as when LGS is significant and MGS is below saturation level, can be used to derive MGS/LGS ratio. Similarly, HGS/MGS ratio can be derived when MGS is significant but HGS is not saturated.

S-NPP VIIRS RTA mirrors suffer significant degradation since the beginning of the mission due to a pre-launch mirror coating problem [3]. It also causes the DNB relative spectral response (RSR) to change due to the differences of degradation rate at different wavelengths. The VCST has analyzed and implemented a methodology of modulated-RSR to address this issue. RSR profiles are adjusted in post-launch for all the RSB and DNB spectral bands. In addition, DNB gains and offsets use the most recent one-year data with a linear fit in forward deliveries.

Specifically, DNB LGS gain calibration is performed for each SD calibration event, where the time-dependent modulated RSR is applied. The MGS and HGS gains are computed based on LGS results, together with the estimated gain ratios of HGS/MGS and MGS/LGS. To maintain $750 \mathrm{~m}$ resolution, DNB has 32 aggregation modes. In all, the DNB calibration is detector, gain-stage, aggregation-mode and HAM-side dependent. 


\section{VIIRS F1 DNB Mode:1 HAM A}

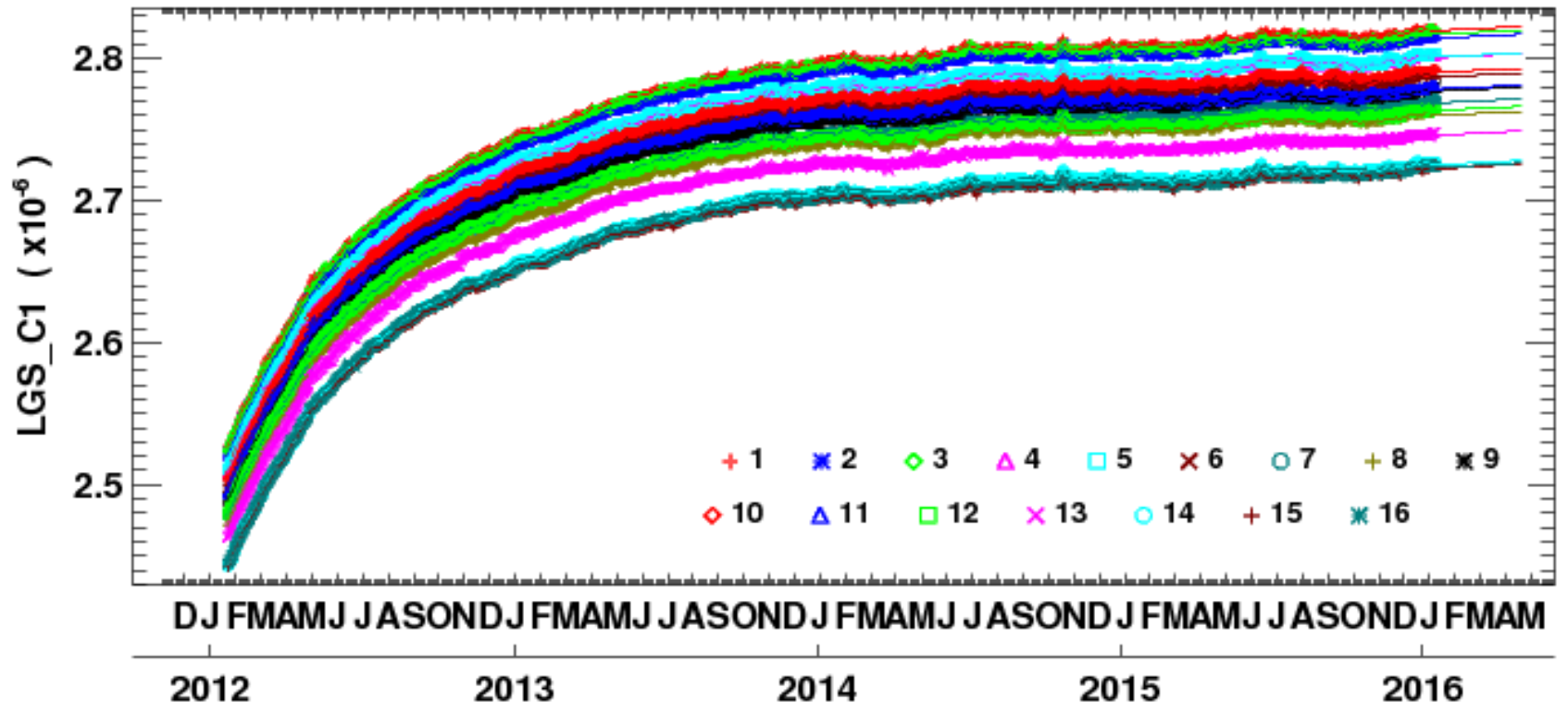

Figure 5 DNB Daily Averaged LGS Gain Trending.

Figure 5 shows the DNB daily averaged LGS gain coefficients (LGS_C1) in units of $\mathrm{W}^{\cdot} \mathrm{cm}^{-2} \mathrm{sr}^{-1}$. In this figure, the aggregation is in the mode-1 (Nadir) case. If normalized at the first point, all DNB detectors are observed with similar trending features in this case. In addition, the last one-year data has been used with a linear fit for forward deliveries. As previously mentioned, the VCST uses the SD signals outside of direct solar illumination to compute the gain ratios between DNB's low-to-mid and mid-to-high gain stage. Figure 6 shows the ratio trend of DNB MGS over LGS using SD signals from aggregation mode-1. This chart presents relatively stable trending over the four-years of operation for all detectors.

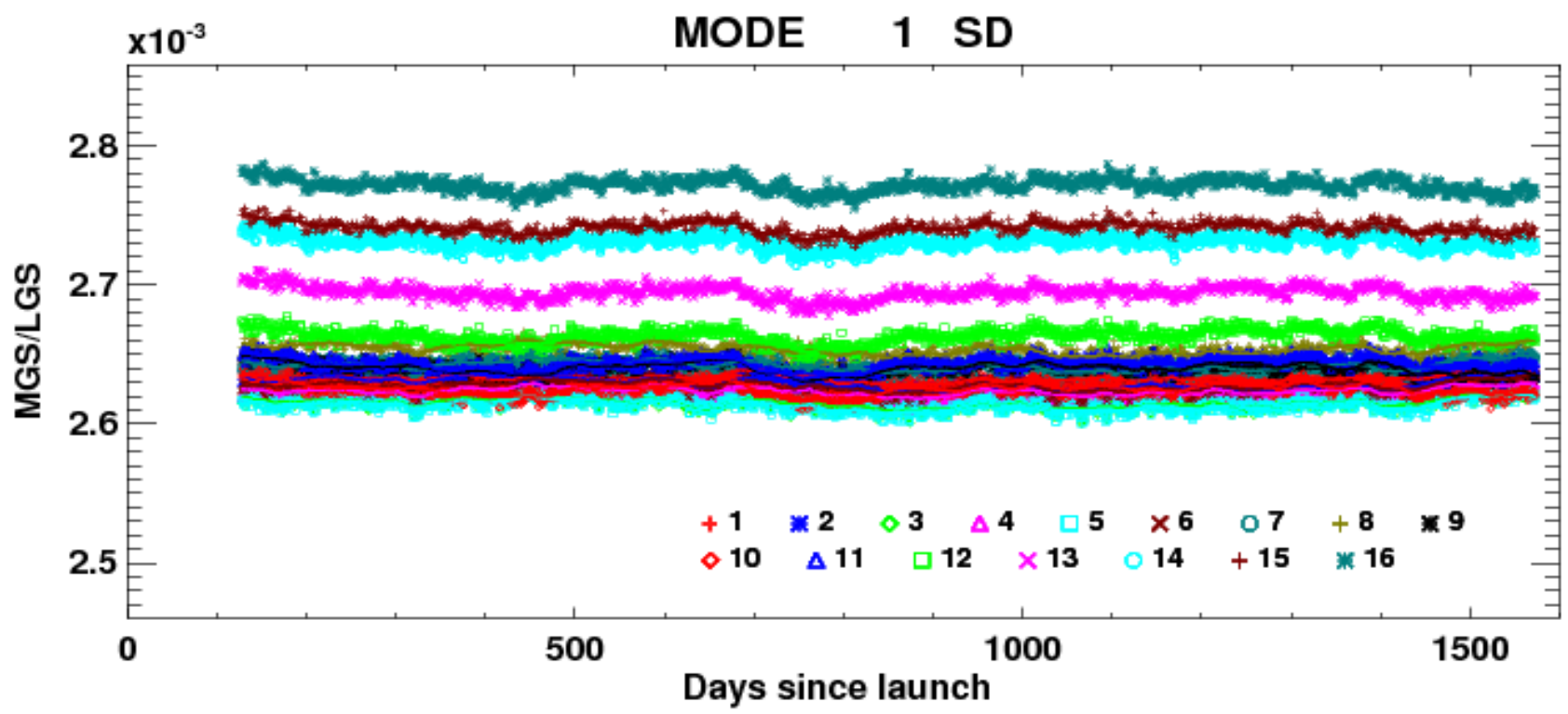

Figure 6 Ratio Trend of DNB MGS over LGS using SD Signals. 
Figure 7 shows DNB daily averaged MGS gain trending in the case of aggregation mode-1 for HAM-side A. This figure is a combination of Figure 5 and Figure 6. Similarly, we can derive HGS gaining trending by using LGS and ratios of MGS/LGS and HGS/MGS.

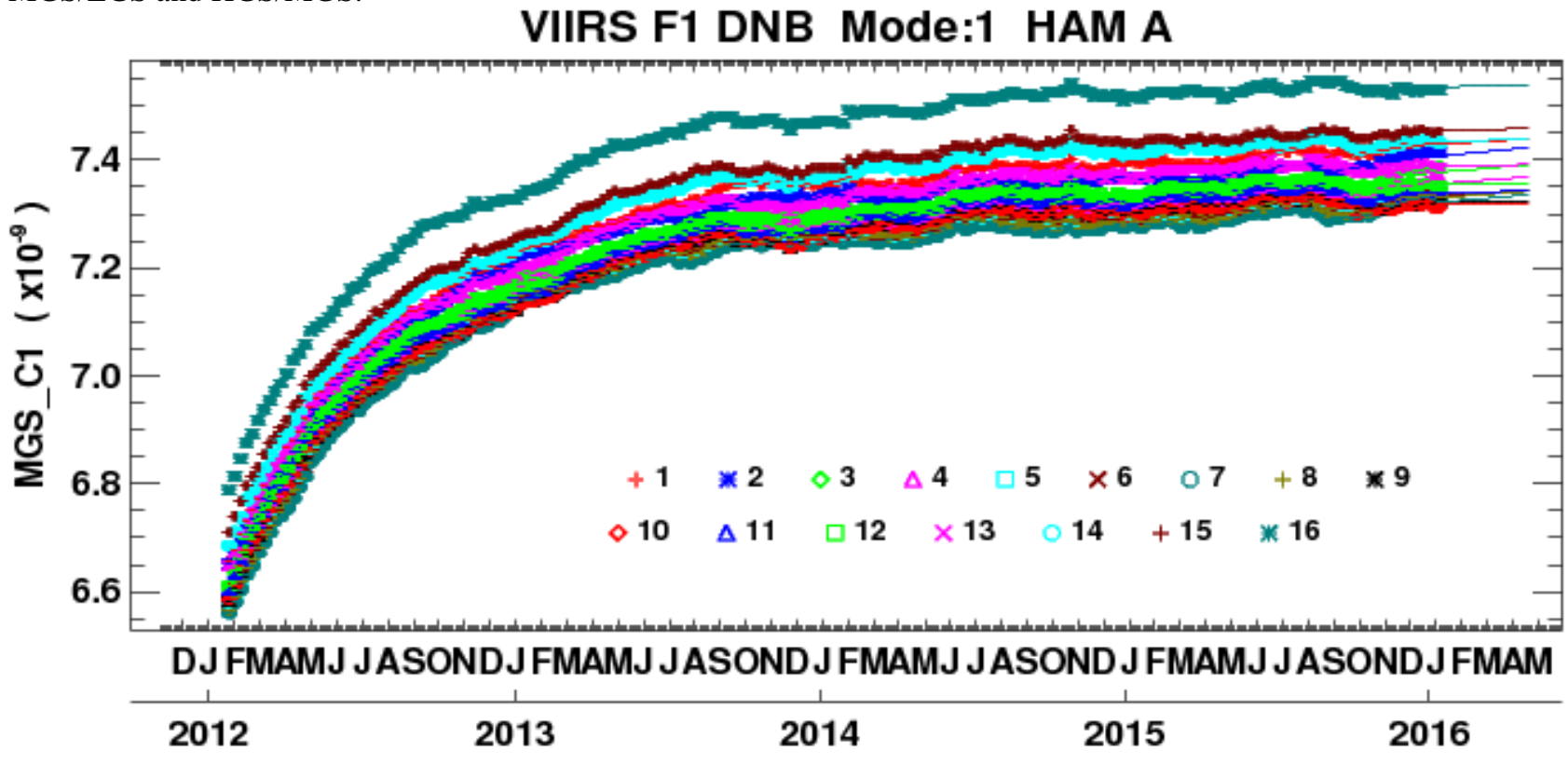

Figure 7 DNB Daily Averaged MGS Gain Trending.

However, due to high sensitivity in HGS sensors, a large fluctuation in the HGS/MGS ratio is expected. Figure 8 shows one example of HGS/MGS ratio for aggregation mode-1. Notice that a downward trend is clearly observed. This downward trend occurs for some modes, but not all. The precise reason is currently unknown. Possibly, it may be the result of HGS sensitivity degradation faster than MGS. We will continue to monitor this behavior.

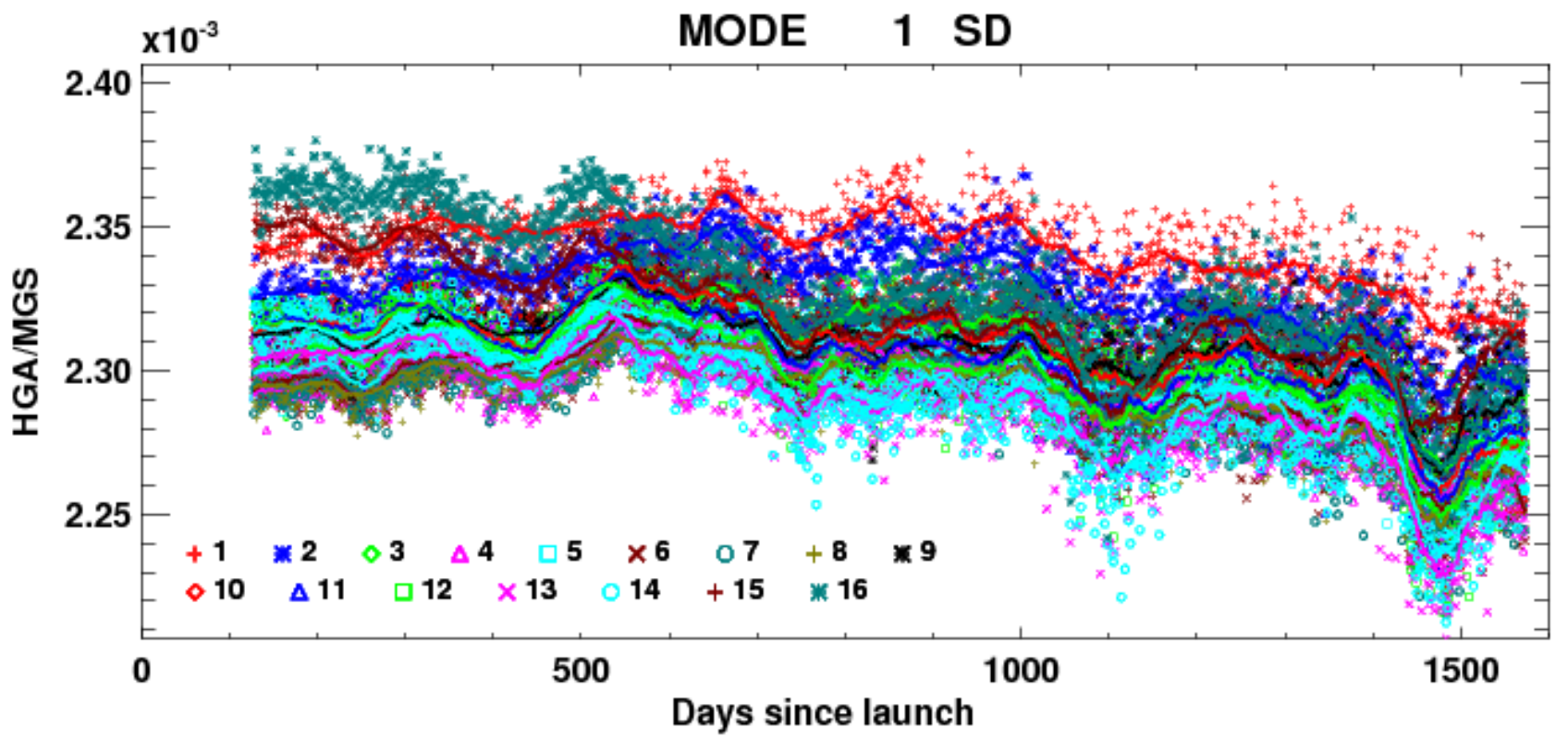

Figure 8 Ratio Trend of DNB HGS over MGS using SD Signals. 


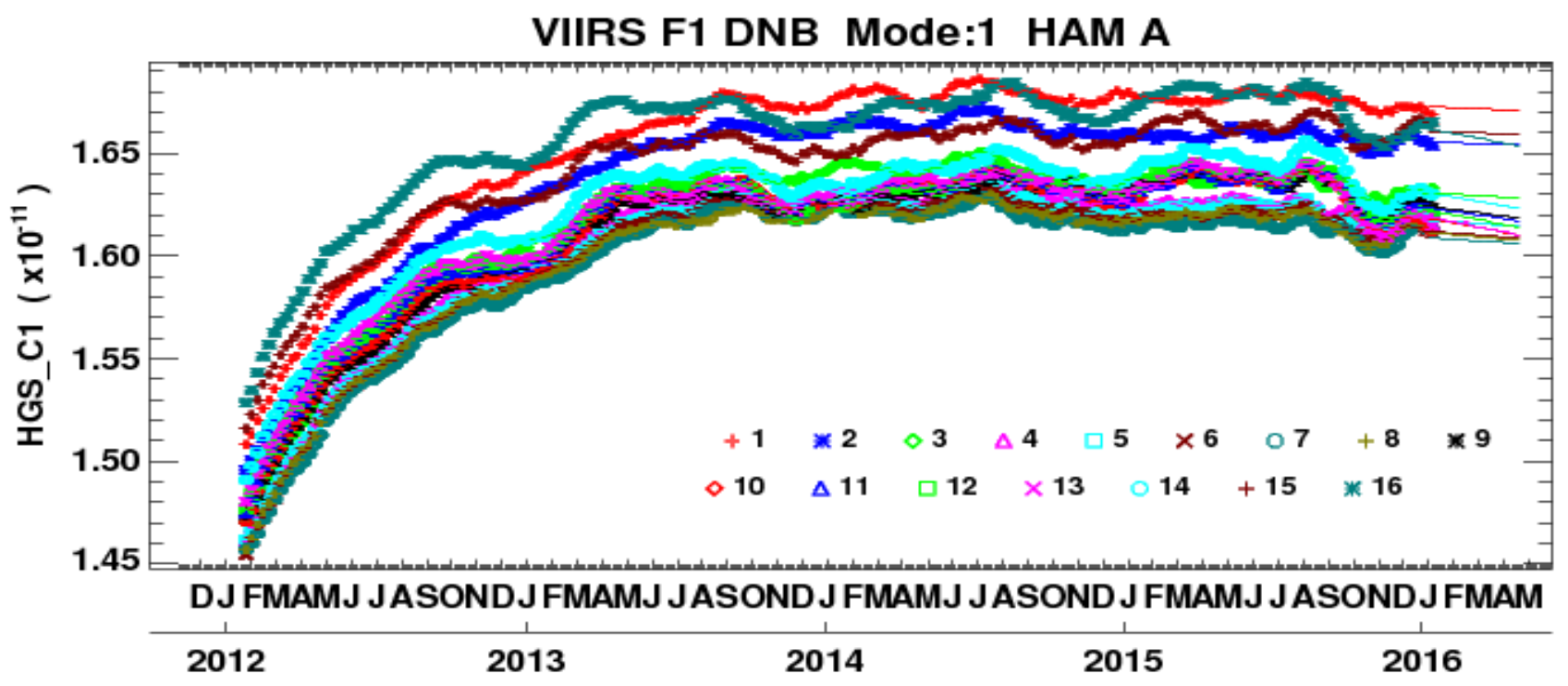

Figure 9 DNB Daily Averaged HGS Gain Trending at Aggregation Mode-1.
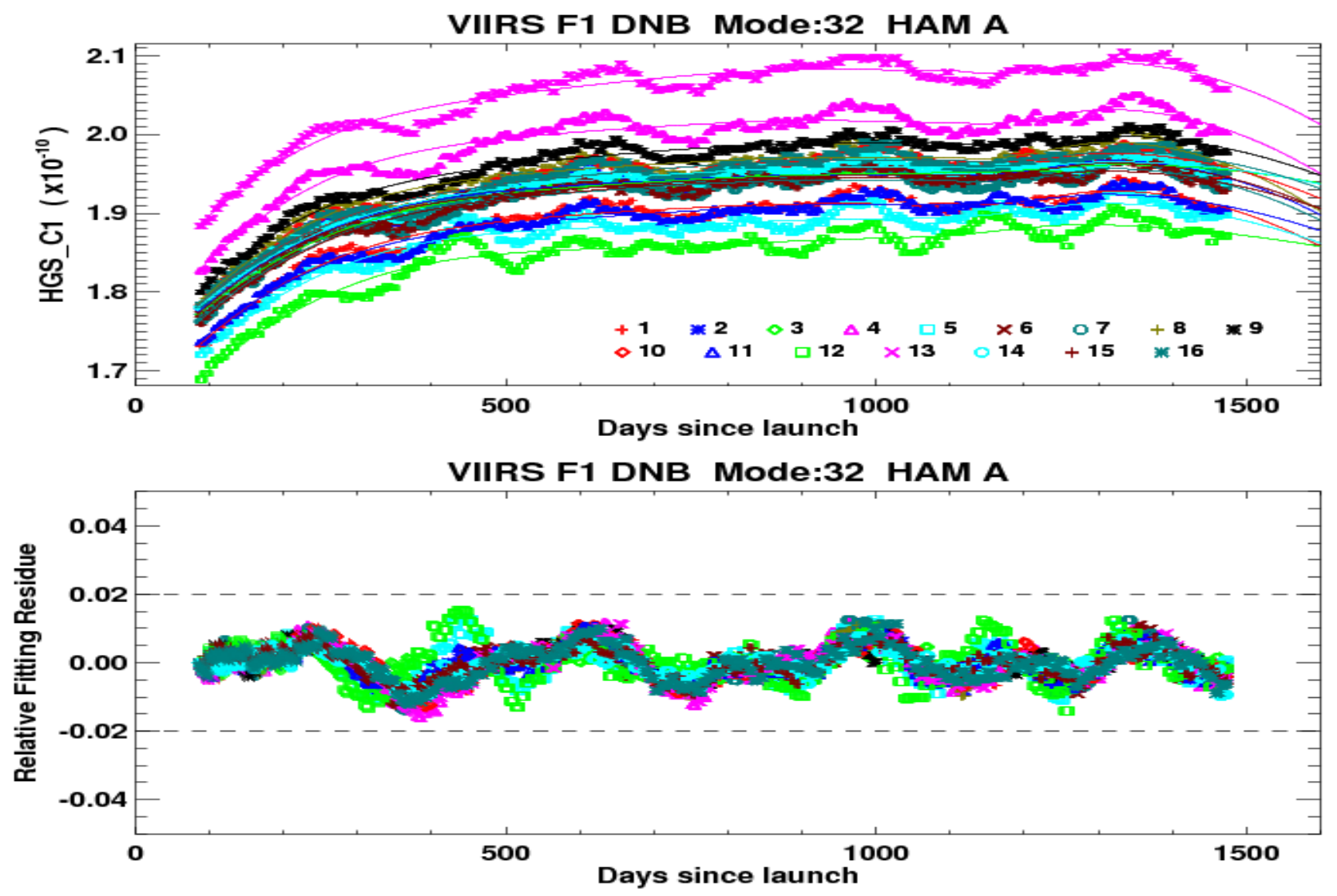

Figure 10 DNB Daily Averaged HGS Gain Trending at Mode-32, with 3-segment Polynomial Fits and Residues.

After applying the ratios of HGS/MGS and MGS/LGS, Figure 9 shows daily averaged DNB HGS gain trending for all 16 detectors. Again, a fitting result is attached to the end of the data for forward deliveries. All detectors have similar 
behavior in the case considered. In practice, the largest fluctuation occurs in the case of HGS aggregation mode-32, whose gain trending is shown on the upper plot of Figure 10. Considering appropriate polynomial fits with multiple segments, the relative fitting residue is shown in the lower plot. There are oscillations observed in the relative fitting residue with a period of approximately one year.

Based on DNB on-orbit trending results, all LGS/MGS/HGS detector gain shares a similar feature of a gradual increase over time. However, the HGS gain trending has a higher variation. This upward trend is mainly due to sensor degradation over time. Since MGS gain is computed from LGS gain and MGS/LGS gain ratios, the MGS gain results indicate that the MGS/LGS ratios are relatively constant in the four years of operation. The HGS gain behavior is dominated by the HGS/MGS gain ratios, which have large uncertainties and yearly oscillations.

\section{STRAY LIGHT CORRECTION}

The S-NPP VIIRS DNB on-orbit stray light impact is much larger and wider than expected in the instrument design stage. It affects up to $25 \%$ of night scenes, where the image quality is significantly degraded. The most persistent stray light contamination is in the mid-to-high latitude regions while the spacecraft is crossing the northern and southern day and night terminators. Quantitative stray light calculations can be performed under new moon conditions, as DNB detectors are with quarter-moon sensitivities. Stray light correction methods and LUTs have been developed and applied to mission-wide SDR reprocess.

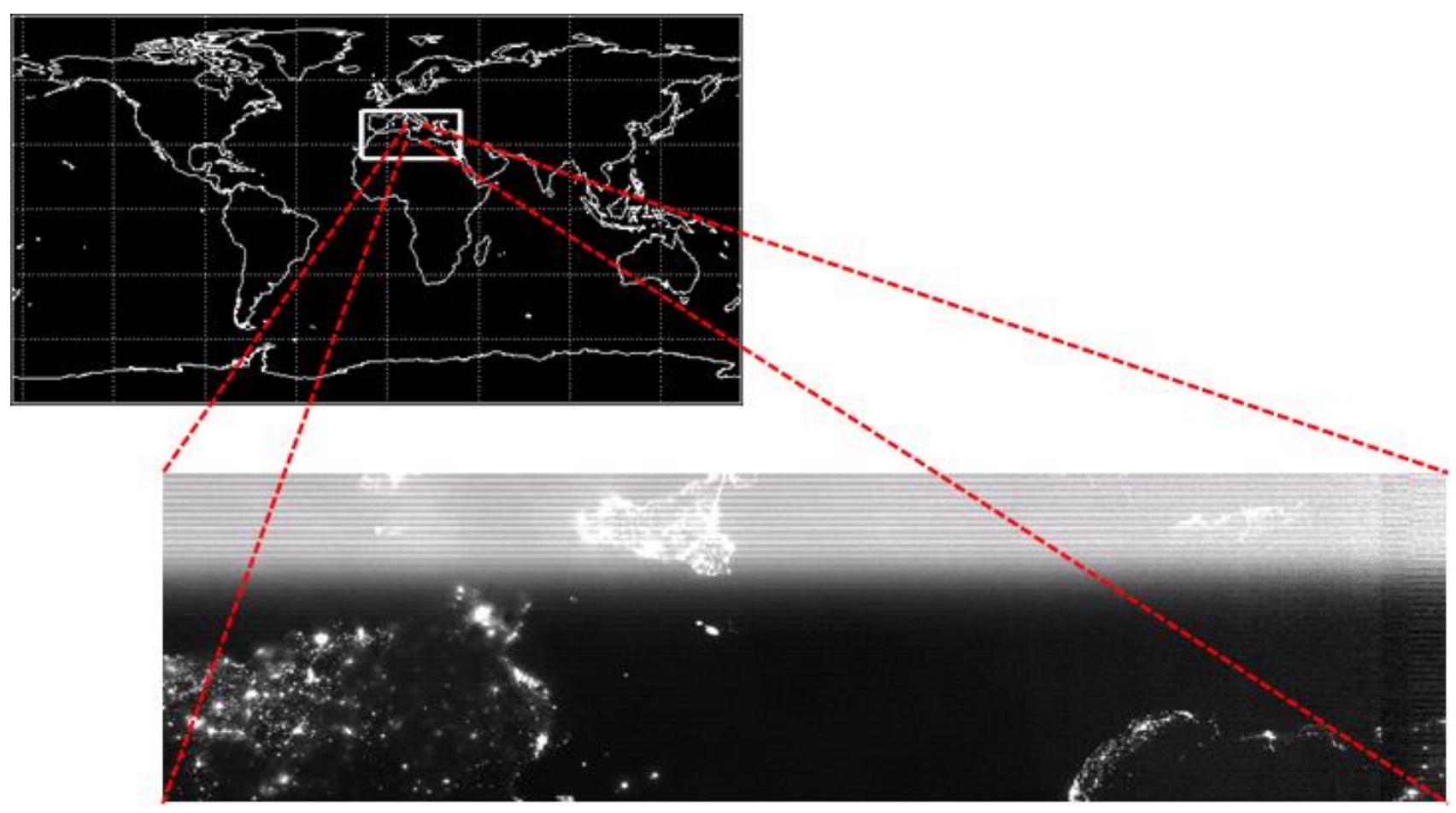

Figure 11 GEO Location Map and DNB Image from July 26, 2014, 1:10 GMT.

In NOAA, the IDPS performs forward processing via on-orbit "AutoCal". As the stray light pattern repeats for yearly Earth-Sun-spacecraft geometry cycle, a stray light correction LUT in the current month is picked from the same month generated in the preceding year. At NASA VIIRS characterization Support Team (VCST), mission-wide stray light correction LUTs are generated and applied in Land PEATE Collection 1.1 reprocessing. Both NASA VCST and NOAA IDPS use the same principles for developing the DNB stray light correction algorithm [4]. The assumption is that small signals observed over the dark Earth scene during a new moon is the result of a combination of stray light and airglow 
[5]. However, there are two major differences between them, such that during a new moon period, the IDPS method uses a whole day dark Earth scene signals (about 14 orbits), where an Earth nighttime light map is applied to exclude nightlights. In VCST, we treat pixels with nightlights as outliers. The reason is that repeat measurements at each successive orbit will cover different area of the Earth. Nightlights are present only on a very small fraction of the Earth's surface. An appropriate outlier exclusion threshold can effectively remove most of the contaminated pixels. The other difference is that we smoothed over the spacecraft zenith and scan angles to compute the dark scene signals, instead of directly averaging the dark scene signals as in the IDPS method. Stray light estimates are derived first by the airglow in the dark scene being subtracted from regions not contaminated by stray light, and then by being subtracted from the dark signals.

Figure 11 shows an example of Europe DNB SDR image before stray light correction. On the top of this figure is a GEO-location map, and on its bottom, the DNB SDR image at 1:10 GMT, July 26, 2014. Obviously, the stray light seriously contaminates the upper area of this nighttime image. The apparent striping is the result of strong stray light detector dependency. Figure 12 shows this image after stray light correction. Without any noticeable quality degradation, this nighttime image presents stray light contamination successfully removed.

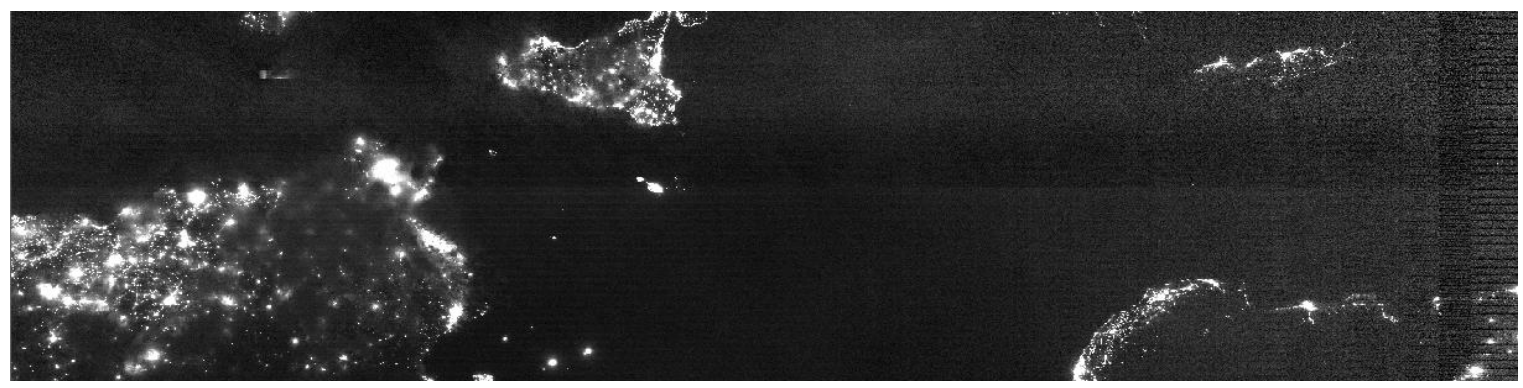

Figure 12 Stray Light Corrected DNB Image from July 26, 2014, 1:10 GMT.

\section{CALIBRATION VALIDATION USING GROUND TARGETS}

To validate DNB calibration performance, images without visible clouds or shadow over Libya-4 and Dome C sites are selected as ground target assessments. The center pixel of the two sites has less than 100 pixel distances to the nadir view (about $75 \mathrm{~km}$ ). 32 by 32 pixels are actually chosen to calculate the DNB mean radiance and reflectance [11]. Images with a standard deviation of radiance higher than $0.0001\left(\mathrm{~W} / \mathrm{cm}^{2} \mathrm{sr}\right)$ are excluded, and data with solar zenith angle higher than 80 degrees are also removed from the Dome $C$ data. The Libya-4 site images are selected from 16-day repeatable orbits so each data has the similar viewing geometry relative to this site. Selected images in the Dome $\mathrm{C}$ site have more overpass orbits with different viewing geometry parameters. The methodology used to characterize the longterm trend for both sites is the same except the BRDF influence. For the Dome C site, the BRDF of the snow is nearly constant inside the DNB spectrum for a specific solar zenith angle, but the reflected radiance in different angular distribution is more isotropic because of atmospheric Rayleigh scattering [12]. A linear BRDF correction is applied to reduce the solar zenith angle and atmospheric Rayleigh scattering influence due to seasonal changes and atmosphere status in Dome C site [11].

The solar irradiance LUT and the modulated RSR LUTs are provided by the VCST. The DNB and M bands SDR produced by the Interface Data Processing Segment (IDPS) and NASA Land Product Evaluation and Algorithm Testing Element (PEATE) are downloaded from Level 1 and Atmosphere Archive and Distribution System (LAADS) (http://ladsweb.nascom.nasa.gov/). We selected both data from nearly nadir overpass for Libya-4 and Dome C since VIIRS launched to the most recent available data in June 2015. The IDPS data is the NOAA-CLASS archived NPPVIIRS data, based on the NOAA official calibration. These calibration LUTs are updated only over time, with no reprocessing over the record. The Land PEATE data has been reprocessed when calibration algorithms are improved. 


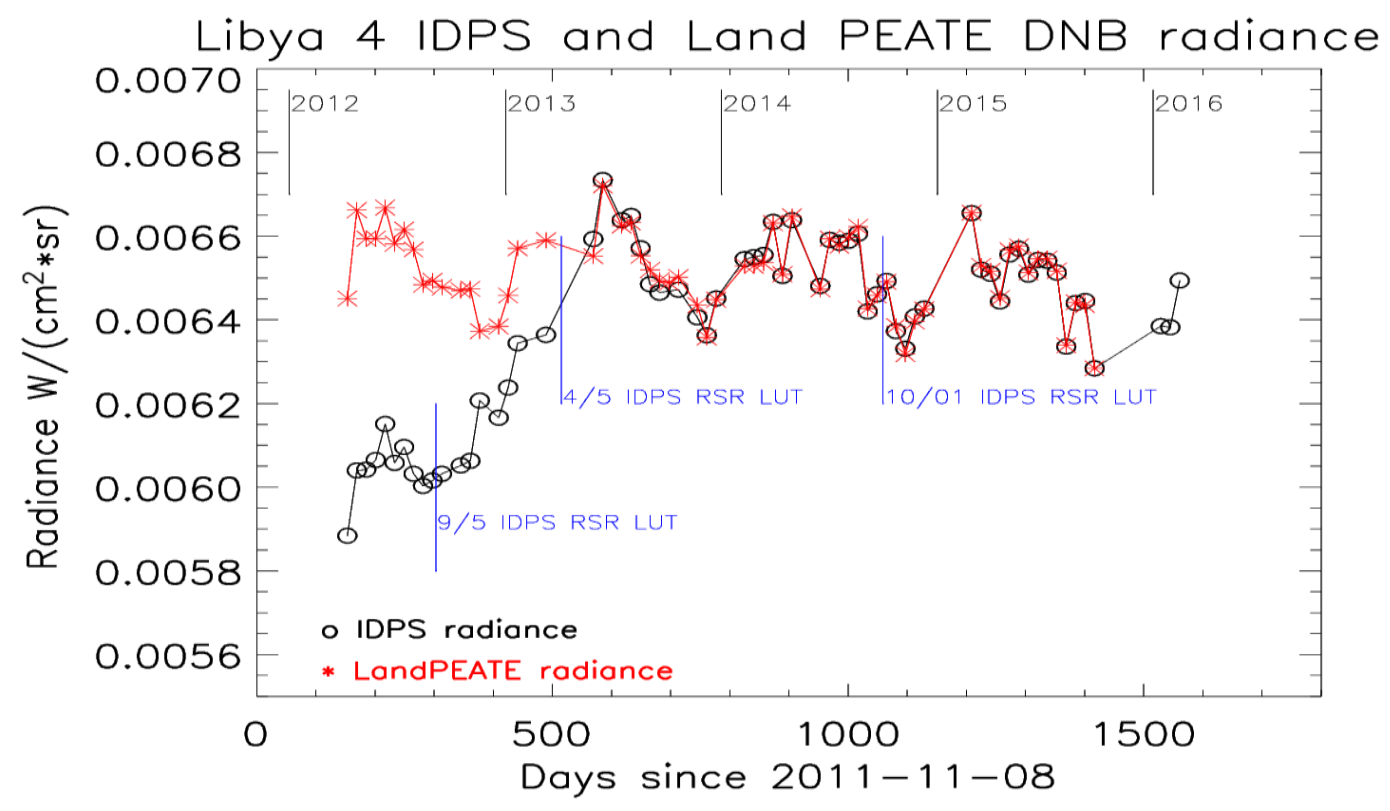

Figure 13 Desert Libya-4 IDPS and Land-PEATE DNB Radiance.

Figure 13 indicates the long-term trends of Libya-4 radiance. Because the LUT updates, the IDPS long-term trends have an upward increase with time (about 14\%-16\% increase by 2014 May 31). The Land PEATE data have a more stable long-term trend due to better quality of LUTs updates and historical data reprocessing. The reflectance of the DNB shows similar trends as the radiance. The noticeable difference in IDPS and Land PEATE trends are caused by the existing inconsistent calibration through the IDPS data processing mission, including the use of SDSM screen transmission and algorithms, and RSR degradation used to compute and update the RSB gain coefficients.

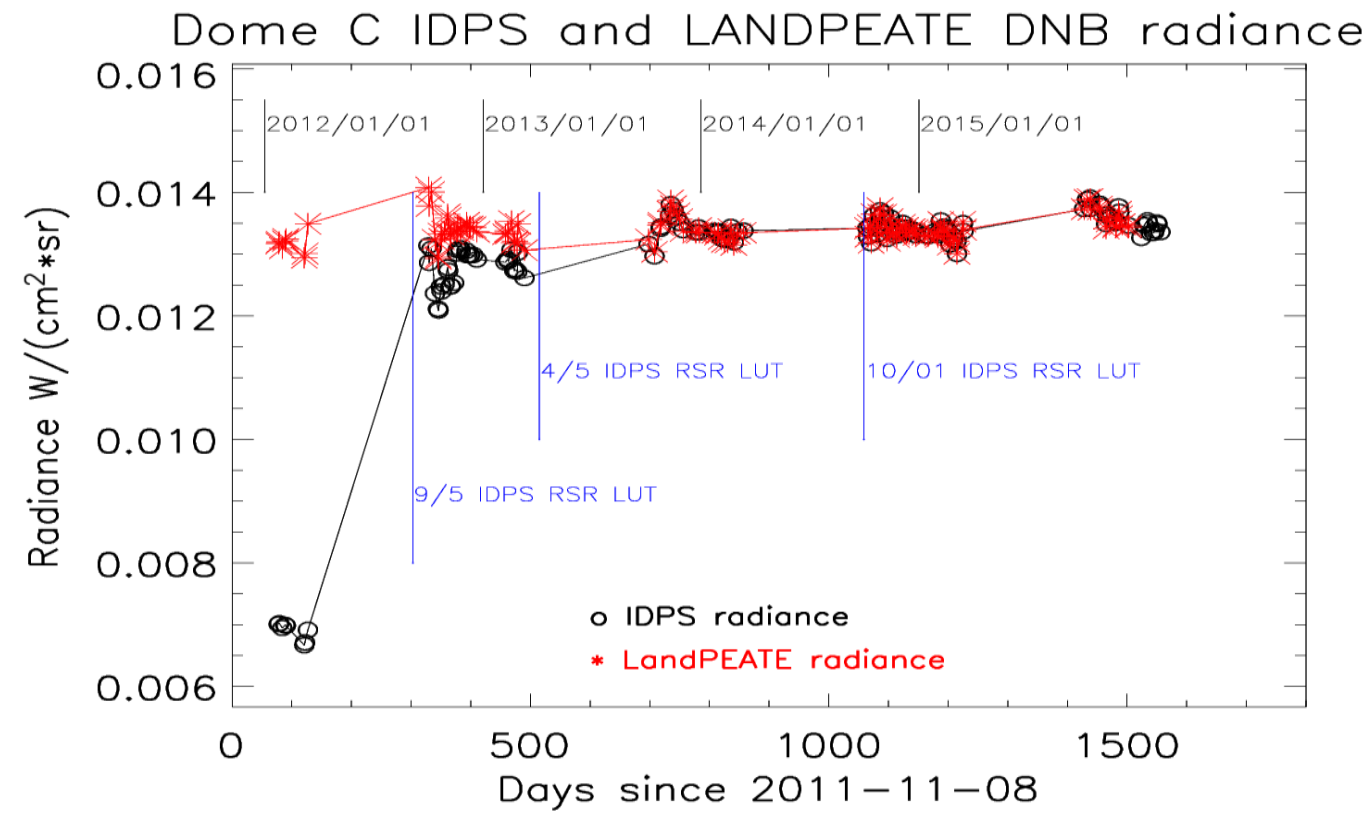

Figure 14 Dome C IDPS and Land-PEATE DNB Radiance.

For Dome $\mathrm{C}$ site radiance, a linear fit for BRDF correction for the Dome $\mathrm{C}$ site Land PEATE data is applied. The data set in 2012 is used as linear fitting training. Each mean radiance of 32x32 pixels is selected after Earth-Sun distance 
normalization and RSR correction. After BRDF correction, the IDPS and Land PEATE radiance data for Dome C is indicated in Figure 14. The IDPS data before 2013 April 5th have significantly lower values than the Land PEATE data due to the difference of RSR LUTs updates. After that time, the ratio of radiance between IDPS and Land PEATE data is within $2.0 \%$.

\section{CONCLUDING REMARKS}

S-NPP VIIRS DNB calibration with stray-light correction has been successfully performed. This ultra-sensitive DNB calibration is conducted as aggregation mode, detector, gain stage and HAM-side dependent. In this paper, recent DNB on-orbit performance has been presented. The overall on-orbit performance of DNB shows that VIIRS RSB degradation behavior is normal. DNB SD and Moon F-factors are matched well. Both DNB LGS gain and offset have been very stable trending, and the gain has gradually increased over time. DNB HGS gain has the largest fluctuation due to involving ratios of MGS over LGS and HGS over MGS. DNB Stray-light correction results show the effectiveness of the stray light estimation/correction algorithm, which is applied in the Land PEATE and produces the entire mission stray light free DNB SDRs. Calibration validation has been conducted using two ground targets: Libya-4 and Dome C. It demonstrates agreement with the IDPS results after IDPS used the updated RSR LUT in April 2013.

\section{ACKNOWLEDGEMENTS}

The authors would like to thank other members of the VCST for their technical discussions and assistance, especially Dr. Truman Wilson for his helpful comments.

\section{REFERENCES}

[1] J. Geis, C. Florio, D. Moyer, K. Rausch and F. Luccia, "VIIRS Day-Night Band Gain and Offset Determination and Performance", Proc. Of SPIE, vol.8510, 851012, pp.1-12, 2012.

[2] S. Lee, K. Chiang, X. Xiong, C. Sun and S. Anderson, "The S-NPP VIIRS Day-Night Band On-Orbit Calibration/Characterization and Current State of SDR Products", Remote Sensing, 2014, v6, pp12427-12446, ISSN 2072-4292.

[3] K. Rausch, S. Houchin, J. Cardema, G. Moy, E. Haas and F. Luccia, "Automated Calibration of the Soumi National Polar-orbiting Partnership (S-NPP) Visible Infrared Imaging Radiometer Suite (VIIRS) Reflective Solar Bands", Journal of Geophysical Research: Atmospheres, v118, pp.13434-13442, 2013.

[4] N. Lei, B. Buenther, Z. Wang and X. Xiong, "Modeling SNPP VIIRS Reflective Solar Bands Optical Throughput Degradation and Its Impacts on the Relative Spectral Response", Proc. SPIE, vol.8866, paper 55, 2013.

[5] S. Millers, S. Weiss and K. Liang, "VIIRS day/night band (DNB) stray light characterization and correction", Proc. SPIE 2013, doi:10.1117/12.2023107.

[6] C. Cao and Y. Bai, "Quantitative Analysis of VIIRS DNB Nightlight Point Source for Light Power Estimation and Stability Monitoring", Remote Sensing, 2014, v6, pp.11915-11935.

[7] S. Lee, and C. Cao, "Soumi NPP VIIRS Day/Night Band Stray Light Characterization and Correction Using Calibration View Data", Remote Sensing, 2016, v8, pp138; doi: 10.3390/rs8020138.

[8] L.B. Liao, S. Weiss, S. Mills and B. Hauss, "Soumi NPP CIIRS Day-Night Band On-orbit Performance", Journal of Geophysical Research: Atmospheres, v118, pp.12705-12718, 2013.

[9] S. Lee, C. Sun, K Chiang and X. Xiong, "An Overview of NASA VIIRS Day-Night Band (DNB) On-orbit Radiometric Calibration", Proc. SPIE 2014, 9218, doi:10.1117/12.2061912.

[10] S. Mills and S. Miller, "VIIRS Day-Night Band (DNB) Calibration Methods for Improved Uniformity", Proc. SPIE 2014, 9218, doi:10.1117/12.2060143.

[11] X. Chen, A. Wu, X. Xiong, N. Lei, Z. Wang, K. Chiang, "Validation of S-NPP VIIRS Day-Night band and M bands performance using ground reference targets of Libya 4 and Dome C". Proceeding of SPIE 2015, Volume 9607, Earth Observing Systems XX.

[12] Hudson, S.r., Wang, S.G., Brandt, R.E., Grenfell, T.C., and Six, D., "Spectral bidirectional reflectance of Antarctic snow: measurements and parameterization,” Journal of Geophysical Research, 111, D018106 (2006). 